\title{
Spatial Domain Adaptive Transmission Based on Precoding in Cognitive MIMO System
}

\author{
WANG Da-ming, ZHU Shi-lei,Tang Shuai,CUI Wei-jia \\ Institute of Information Engineering, \\ The PLA Information Engineering University, \\ Zhengzhou, China, \\ zhushilei3620@163.com
}

\begin{abstract}
In cognitive radio system, secondary users are difficult to obtain the CSI (channel state information) of the interference channel from the primary users which have absolute right of the spectrum sharing with secondary users due to the unlicensed utilization of spectrum resource. In order to guarantee the transparency of secondary transmission to primary transmission, a so-called "Detect-avoid" transmission mechanism is adopted in traditional cognitive radio system to avoid interference to primary user. However, it performs only in time-domain with inevitable underutilization of spectrum opportunities. In this paper, MIMO technique is introduced for secondary users to detect the real-time state information of the interference channel with its own cognitive capability and to a just the precoding matrix adaptively using the detection results, so that orthometric transmission with primary users in spatial domain is realized. Simulation result shows that the performance of the proposed algorithm without the knowledge of interference channel's CSI can approach to precoding algorithm based on perfect CSI.
\end{abstract}

Keywords-Cognitive MIMO; subspace decomposition; transmission mechanism; precoding

\section{INTRODUCTION}

Because of the user's dynamic, the utilization rate of spectrum which is staticly distributed by traditional mechanism is very low [1]. Therefore, the cognitive radio (CR) which could use the resource of spectrum according to the electromagnetic environment emerged. Cognitive user (CU) finds the spectrum which is idle by detecting the environment, then completes the communication with the idle spectrum. But as an unauthorized user, the CU should detect the channel periodically to seek the idle spectrum. If the authorized user (PU) occupies the spectrum resource, the $\mathrm{CU}$ should release the spectrum immediately to avoid the interference to the PU, which is called "detect-avoid" transmission mechanism. However, the mode based on "detect-avoid" will waste a part of the spectrum and the quality of communication is hard to be guaranteed [2].

Communication is a procedure which makes multipurpose use of many facts such as time, power, space and frequency spread code [3], so it is feasibility to taking the opportunity of access by spreading the dimension of the resource. The MIMO system attracts the researchers' attention due to the high efficiency of transmission and reliability of the link [6] in recent years. In the literature [2], the performance of communication is improved a lot by using an access mode based on space division multiplexing with the technology of space signal processing. The MIMO' capacity is proposed by combining information theory in the literature [4]. With the technology of precoding, the interference from the $\mathrm{CU}$ to the PU is effectively restrained or eliminated in the literature [5-9], which would lead to a good system performance, besides, satisfy the restrict of the interference. However, in the literature above-mentioned, there is a hypothesis that the CU gets the knowledge of CSI (channel state information). At the same time, the CU and the PU belongs to different communication systems, as the authority of the frequency spectrum, it is not necessary for the PU to feed back the CSI to the CU, so it is hard to get the CSI in practice.

In reality, the CU has the ability of self-adaption which can get the channel parameters by monitoring the channel of the PU and detecting the channel, thus modifying the transmission strategy, to get the optimal system performance on the premise of pledging the PU's QoS. On account of the considerations above, a transmission mechanism based on subspace decomposition in cognitive MIMO system is proposed in this paper by combining the technology of MIMO and traditional transmission mechanism. During the period of channel detecting, the space dimension of the signal transmitted by the PU is estimated exactly by the criteria based on MDL (minimum description length) for the CU which could get signal space and noise space by taking subspace decomposition on the covariance matrix detected. During the transmission stage, taking the orthogonality between signal space and noise space, the CU projects the signal into the PU' noise space by precoding, which could avoid the interference to the PU, then takes SVD (singular value decomposition) on the matrix of the CU' channel, which could improve the system performance by the distribution mode of power-injection.

\section{SYSTEM DESCRIPTION}

Taking account of the scene, which the CU and the PU sharing the channel, is shown in figure 1 . The cognitive system is consist of a sender (CR-Tx) (takes $n_{T}$ transmitter antennas)and a receiver (CR-Rx) (takes $n_{R}$ receiving antennas), that there is no competition and interference between the cognitive users. The PU system is consist of a 
couple of the PU, which are equipped with $n_{1}$ and $n_{2}$ antennas. Assuming that the CU system and the PU system uses time-division-duplex (TDD) mode, and the channel meets the reciprocal theorem. Assuming that the channel between the PU which is called $H_{1}$ (or $H_{1}^{H}$ ) and the channel between the $\mathrm{CU}$ which is called $H$ all are Rayleigh fading channel, $G_{i}(i=1,2,3,4)$ are the channels that the PU and the CU could interfere with each other.

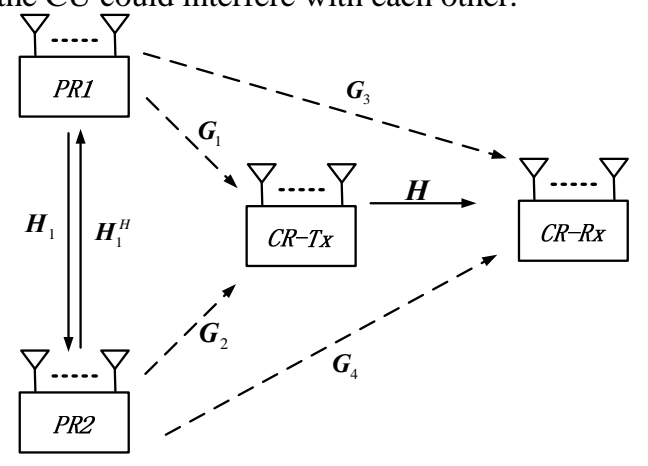

Figure 1. Model of the CU system and PU system sharing the spectrum

Assuming that the signal send by the $\mathrm{CU}$ is vector $X$, which is l-dimensional, and the relevant precoding matrix is $F$, which is $n_{T} \times l$-dimensional, the decoding matrix at the receiving node is $W$, which is $l \times n_{R}$-dimensional, and the signal send by the PU is vector $s_{j}(j=1,2)$, then the signal received by the CU and PU are shown below.

$$
\left\{\begin{array}{l}
\boldsymbol{y}_{R}(n)=\boldsymbol{W}\left[\boldsymbol{H} \boldsymbol{F} \boldsymbol{x}(n)+\boldsymbol{G}_{3} \boldsymbol{s}_{1}(n)+\boldsymbol{n}_{R}(n)\right] \\
\boldsymbol{r}_{2}(n)=\boldsymbol{H}_{1} \boldsymbol{s}_{1}(n)+\boldsymbol{G}_{2}^{H} \boldsymbol{F} \boldsymbol{x}(n)+\boldsymbol{z}(n)
\end{array}\right.
$$

$I_{C R}=G_{3} S_{1}(n)$ is the interference to the $\mathrm{CU}$ that is caused by the PU, $I_{P R}=G_{2}^{H} F x(n)$ is the interference to the PU that is caused by the CU, which is ignored or dealt as noise in most literatures, but in practice the PU system and the CU system would interference with each other, so it is needed to take into account when designing the precoding. $n_{R}(n)$ and $z(n)$ are additive white noises in the channel of the $\mathrm{CU}$ and PU.

\section{TRANSMission MECHANISM BASED ON SUbSPACE DECOMPOSITION}

Fig. 2 shows the basic structure of the radio frame, which is consist of two parts, one is the section of channel detecting, the other is data transmission. As the limited of half-duplex of the antenna in the user terminal, channel detecting and data transmission should not work at the same time. During the period of channel detecting, the CU should keep silence, and look for the idle spectrum by monitoring the channel. Once the PU emerges again, the CU should be aware of it and release the resource immediately. Considering the fact that the traditional "detect-avoid" transmission mechanism should waste a part of the spectrum resource, on the foundation of the traditional structure of radio frame, this paper proposes a transmission mechanism based on subspace decomposition by combining MIMO's spatial processing ability.

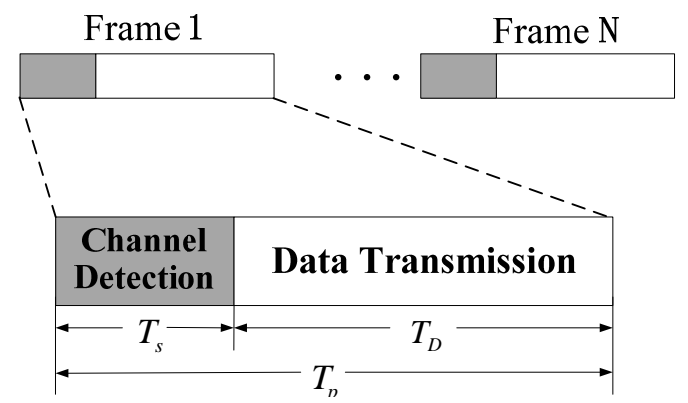

Figure 2. The structure of radio frame

\section{A. The Section of Detecting}

Assuming the PU works as TDD model, the signal sent by the PU i should be described as follow.

$s_{i}(n)=\left\{\begin{array}{cc}\tilde{s}_{i}(n) & \text { the signal sent by the PU i }(i=1,2) \\ 0 & \text { else }\end{array} n=1,2, \cdots, L\right.$

$\widetilde{S}_{i}(n)$ are independent and identically distributed fake randomicity signals [9], which covariance matrix is $\sigma_{s}^{2} I$. L is the snapshots during the channel detecting. Assuming that the probability PU $i$ sends signal is $\alpha_{i}\left(\alpha_{1}+\alpha_{2}=1\right)$, then the covariance matrix received is $R_{s i}=E\left(s_{i}(n) s_{i}^{H}(n)\right)=\alpha \sigma_{s}^{2} I$.

When the PU1 (or 2) sends signal, the signals received by the CU sender and receiver are as follow.

$\left\{\begin{array}{l}\boldsymbol{y}_{1}(n)=\boldsymbol{G}_{1} \boldsymbol{s}_{1}(n)+\boldsymbol{n}_{T}(n) \\ \boldsymbol{y}_{2}(n)=\boldsymbol{G}_{3} \boldsymbol{s}_{1}(n)+\boldsymbol{n}_{R}(n)\end{array}\right.$ (or) $\left\{\begin{array}{l}\boldsymbol{y}_{3}(n)=\boldsymbol{G}_{2} \boldsymbol{s}_{2}(n)+\boldsymbol{n}_{T}(n) \\ \boldsymbol{y}_{4}(n)=\boldsymbol{G}_{4} \boldsymbol{s}_{2}(n)+\boldsymbol{n}_{R}(n)\end{array}\right.$

then the corresponding covariance matrix are as follow.

$$
\boldsymbol{R}_{j}=E\left[\boldsymbol{y}_{j}(n) \boldsymbol{y}_{j}^{H}(n)\right]=\alpha_{j} \sigma_{s}^{2} \boldsymbol{G}_{j} \boldsymbol{G}_{j}^{H}+\sigma_{n}^{2} \boldsymbol{I}
$$

To keep the commonality, take $R_{1}$ as an example, making eigenvalue decomposition on $R_{1}$.

$$
\boldsymbol{R}_{1}=\boldsymbol{U}_{1} \boldsymbol{\Lambda}_{1} \boldsymbol{U}_{1}^{H}=\boldsymbol{U}_{s 1} \boldsymbol{\Lambda}_{s 1} \boldsymbol{U}_{s 1}^{H}+\sigma_{n}^{2} \boldsymbol{U}_{n 1} \boldsymbol{U}_{n 1}^{H}
$$

$U_{s 1}$ is $n_{T} \times d$-dimensional eigenvector of the signal subspace, which is consist of eigenvectors corresponding to $d$ bigger eigenvalues of the covariance matrix. $U_{n 1}$ is $n_{T} \times\left(n_{T}-d\right)$-dimensional eigenvector of the noise subspace, which is consist of eigenvectors corresponding to $n_{T}-d$ smaller eigenvalues. According to the subspace decomposition theory [11], $G_{1}$ and $U_{n 1}$ is orthogonal ( $G_{1}^{H} U_{n 1}=0$ ), that means $U_{n 1}$ spans the orthogonal space of the matrix $G_{1}$ of the interfered channel. Therefore, if the $\mathrm{CU}$ projects the signal into the noise space, the interference 
to the PU should be avoided. So, orthogonal transmission between the PU and CU is realized by using noise space. Accordingly, it should avoid the interference from the PU by using $U_{n 3}$ (or $U_{n 4}$ ).

Taking account of the fact that the data matrix received is finite-length, the maximum likelihood estimation of $R_{1}$ is as follow.

$$
\hat{\boldsymbol{R}}_{1}=\frac{1}{L} \sum_{n=1}^{L} \boldsymbol{y}_{1}(N) \boldsymbol{y}_{1}^{H}(N)
$$

Making eigenvalue decomposition on $\hat{R}_{1}$, the result shows as follow.

$$
\hat{\boldsymbol{R}}_{1}=\hat{\boldsymbol{U}}_{1} \hat{\boldsymbol{\Lambda}}_{1} \hat{\boldsymbol{U}}_{1}^{H}=\hat{\boldsymbol{U}}_{s 1} \hat{\boldsymbol{\Lambda}}_{s 1} \hat{\boldsymbol{U}}_{s 1}^{H}+\hat{\boldsymbol{U}}_{n 1} \hat{\boldsymbol{\Lambda}}_{n 1} \hat{\boldsymbol{U}}_{n 1}^{H}
$$

$\hat{\Lambda}_{1}=\operatorname{diag}\left(\lambda_{1}, \lambda_{2}, \cdots \lambda_{n_{T}}\right)$ is descending sort of the eigenvalues. The eigenvalues are unequal with each other under the non-ideal conditions, so it is difficult to estimate the number of the space dimension of the signal sent by the PU, we should calculate the dimension $d$ by using the MDL rule of source estimation theory.

$$
\begin{gathered}
M D L(k)=L(m-k) \ln \left(\frac{\frac{1}{m-k} \sum_{i=k+1}^{m} \hat{\lambda}_{i}}{\prod_{i=k+1}^{m}\left(\hat{\lambda}_{i}\right)^{1 /(m-k)}}\right)+\frac{1}{2} k(2 m-k) \ln L \\
d=\arg \min (\operatorname{MDL}(k))
\end{gathered}
$$

Thereby calculate the noise space $\hat{U}_{n 1}$. Accordingly, we should get the noise space $\hat{U}_{n 2}, \hat{U}_{n 3}$ and $\hat{U}_{n 4}$ corresponding $G_{2}, G_{3}$ and $G_{4}$.

\section{B. The Section of Data Transmission}

To decrease the time of transmission, the CU should maximize the throughput, which would reduce the probability of collision with the PU, and improve the spectrum utilization.

Firstly, to avoid the interference between the PU and $\mathrm{CU}$, the CU must project the signal into the orthogonal space of the interfered channel, which would avoid the interference ( $I_{P R}$ ) to the PU. Multiply $\hat{U}_{n 2}$ at the right of the channel $H$, and multiply $\hat{U}_{n 3}^{H}$ at the left to eliminate $I_{C R}$, so the equivalent channel is $H_{e q}=\hat{U}_{n 3}^{H} H \hat{U}_{n 2}$. Taking singular value decomposition (SVD) on it, the result is as follow.

$$
\boldsymbol{H}_{e q}=\boldsymbol{U} \boldsymbol{\Sigma} \boldsymbol{V}^{H}
$$

As $\Sigma=\operatorname{diag}\left(\sqrt{\lambda_{1}}, \sqrt{\lambda_{2}}, \cdots, \sqrt{\lambda_{k}}\right), k$ is the number of the parallel sub-channels. We should distribute $P$ into the $k$ parallel sub-channels to realize the best power allocation by using power-injection theorem. As $\boldsymbol{P}_{i}=\left(v-1 / \lambda_{i}\right)^{+}, i=1, \cdots, k$ [6], then the best precoding matrix is as follow.

$$
\boldsymbol{F}=\hat{\boldsymbol{U}}_{n 2} \boldsymbol{V} \operatorname{diag}\left(\sqrt{P_{1}}, \sqrt{P_{2}}, \cdots, \sqrt{P_{k}}\right)
$$

The corresponding decoding module is as follow.

$$
\boldsymbol{W}=\boldsymbol{U}^{H} \hat{\boldsymbol{U}}_{n 3}^{H}
$$

To conclude, the process of the mechanism based on subspace decomposition is shown in Fig. 3.

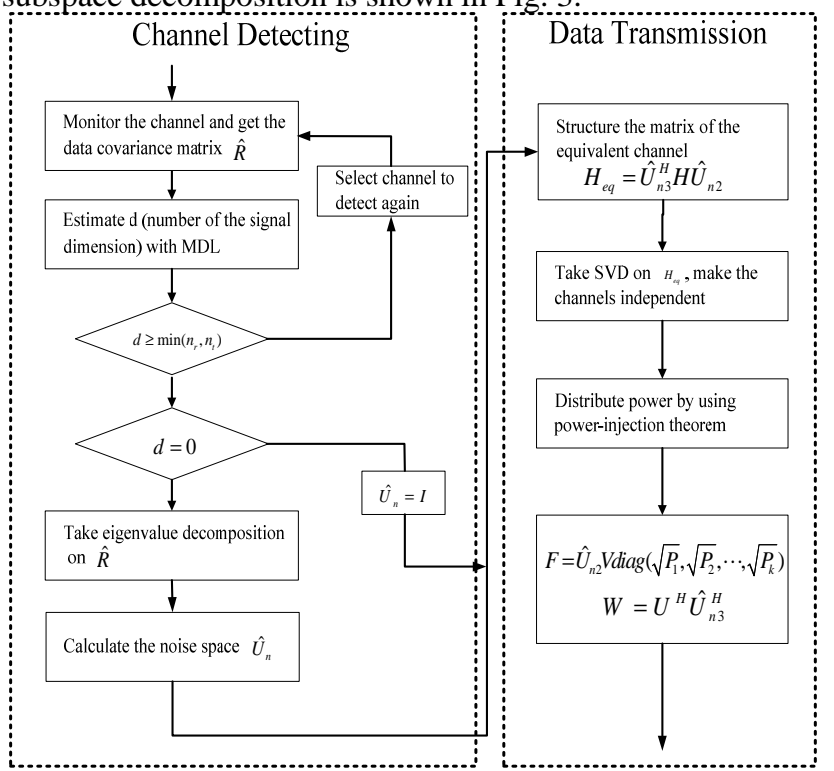

Fig. 3 The process of the mechanism based on subspace decomposition

\section{Analysis of Simulation Experiment}

To verify this algorithm, we had made simulation about this algorithm. Assuming that the antenna configuration of the PU meets $n_{1}=n_{2}=2$, the antenna configuration of the CU meets $n_{T}=n_{R}=5$, the signal is modulated as QPSK. Without channel coding, the launching power is 1 . We made 10000 simulation by using Monte Carlo theory, and the length of the data sent by the CU was 7200bit.

Fig. 4 shows the different performance of detecting by using MDL criterion at the different snapshots. As the same snapshots, the correct detection probability shapes as S with the change of the signal SNR received by the CU, and the performance becomes stable at about $7 \mathrm{~dB}$. When snapshots is little, the estimate effect is relatively poor, that is because the eigenvalues are dispersive. With the increasing of snapshots, the level of the noise eigenvalues become low, so the correct detection probability rises.

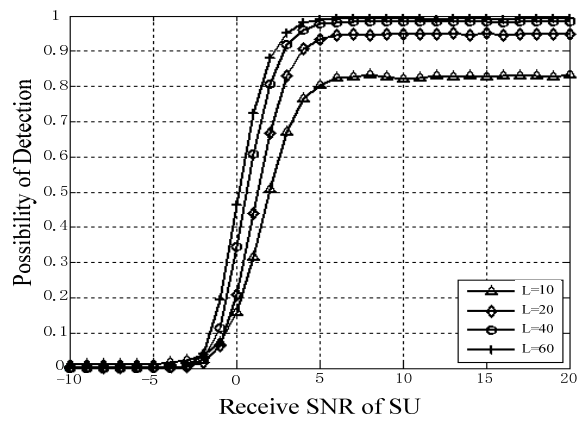


Fig. 4 Performance of detecting by using MDL criterion at the different snapshots

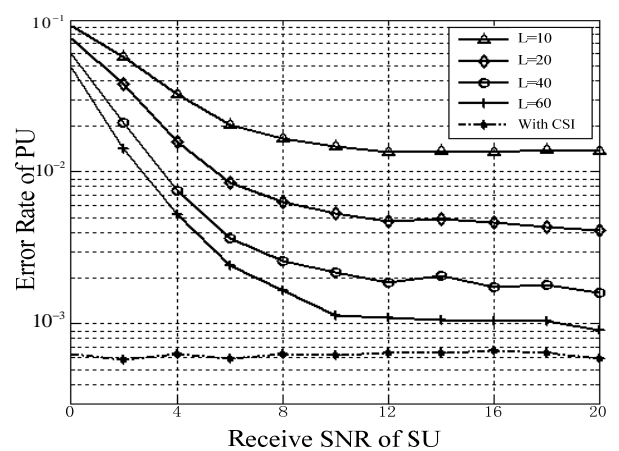

Fig. 5 Error rate of the PU

Fig. 5 shows the curves of error rate calculate by this algorithm when the CU knows or not knows the CSI of interference channel, as the SNR received by the PU is $15 \mathrm{~dB}$. The result shows with the limit of the snapshots and the SNR, the noise space estimated is not good, which would make a great influence on the communication quality. At the same time, the $\mathrm{CU}$ could decrease the interference to the PU greatly by increasing the snapshots and the SNR, which would make the error rate performance at the condition that the CSI is unknown as good as at the ideal condition of the CSI is known.

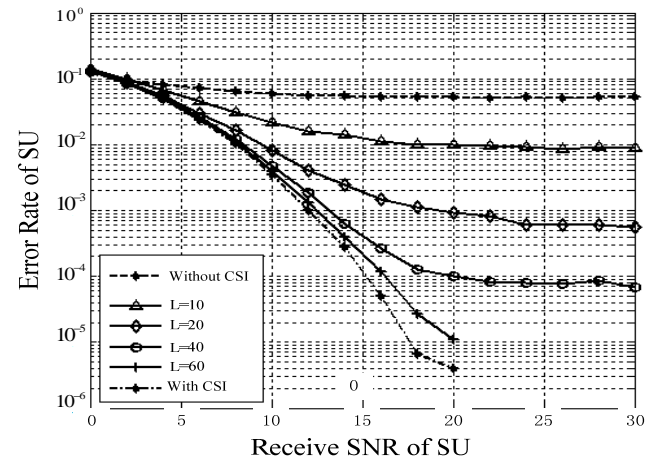

Fig. 6 Error rate performance of the CU at different snapshots

Fig. 6 shows the curve of the error rate changing with the SNR received by the $\mathrm{CU}$. When deal the interference of the $\mathrm{PU}$ as noise, the interference to the $\mathrm{CU}$ may be great (shown as dash line in the figure ), so we need further process of space orthogonalization. With the increase of the snapshots, the performance of the $\mathrm{CU}$ improves, tending to the ideal transmission when the CSI is known.

\section{CONCLUSION}

The " detect-avoid" transmission mechanism in traditional cognitive radio system is difficult to guarantee the communication quality of cognitive user (CU). This paper proposes a transmission mechanism based subspace decomposition by combining MIMO's spatial processing ability. We would realize the adaptive allocation of the degree of space freedom by adjusting the signal space and getting the spatial information of the PU system through detecting the Second-Order Statistics of the PU. The simulation result shows that the performance of the proposed algorithm without the knowledge of interference channel' $s$ CSI can approach to precoding algorithm based on perfect CSI on the condition that snapshots is hyper-60.

\section{REFERENCES}

[1] Krenik W, Batra A. Cognitive radio techniques for wide area networks [C]. Design Automation Conference (DAC’05), 2005: 409412.

[2] Li Zhao, Zhao Lin-jing, Liu Qin. Space Division Multiplexing Based Opportunistic Spectrum Access in Cognitive Radio Network[J]. Journal of Electronics \& Information Technology, 2011, 33(5): 11721177.

[3] Vuk M, Jose S, Xavier R, et al. Resource modeling for a joint resource management in cognitive radio [C]. IEEE International Conference on Communications, Beijing, 2008: 4175-4180.

[4] Sridharan S, Vishwanath S. On the capacity of a class of MIMO cognitive radios, IEEE Journal of Selected Topics in Signal processing, 2008, 2(1):103-117.

[5] Zhang R, Liang Y C. Exploiting multi-antennas for opportunistic spectrum sharing in cognitive radio networks [J]. IEEE Journal of Selected Topics in Signal Processing, 2008, 2(1): 88-101.

[6] Devroye N, Vu M, Tarokh V. Cognitive ratio networks[J]. IEEE Signal Processing Magazine, 2008, 25(6): 12-23.

[7] Zhang L, Xin Y, LIANG Y C. Weighted sum rate optimization for cognitive radio MIMO Broadcast Channels[J]. IEEE Transactions on wireless communication. 2009, 8(6): 2950- 2959.

[8] Zhang C H, Guo L. BD Precoding schemes for cognitive MIMO system [J]. Network Infrastructure and Digital Content, 2009: 11-15.

[9] Lee K J, Lee K. MMSE Based Block Diagonalization for Cognitive Radio MIMO Broadcast Channels [J]. IEEE Transactions on wireless communication, 2011: 1-4.

[10] Rong Mei, Zhu Shi-hua, Li Feng. Spectrum Sharing Based on Frobenius Norm in Cognitive Radio Networks [J]. ACTA Electronica Sinica, 2011, 31(1): 95-100.

[11] $\mathrm{Xu} \mathrm{z.} \mathrm{Perturbation} \mathrm{analysis} \mathrm{for} \mathrm{subspace} \mathrm{decomposition} \mathrm{with}$ application in subspace-based algorithms[J]. IEEEE Trans. Sig. Proces. 2002, 50(11): 2820-2830.

[12] $\mathrm{Xu} \mathrm{z}$. On the second-order statistics of the weighted sample covariance matrix[J]. IEEE Trans. Sig. Proces. 2003, 51(2): 527-534.

[13] Zhang Xian-da. Matrix Analysis and Applications[M]. Tsinghua University Press, 2004. 